\title{
miR-130a acts as a potential diagnostic biomarker and promotes gastric cancer migration, invasion and proliferation by targeting RUNX3
}

\author{
HONG JIANG ${ }^{1}$, WEI-WEI YU ${ }^{1}$, LU-LU WANG ${ }^{2}$ and YANG PENG ${ }^{1}$ \\ ${ }^{1}$ Department of Geriatrics, Tongji Hospital, Tongji Medical College, Huazhong University of Science and Technology, \\ Wuhan, Hubei 430030; ${ }^{2}$ Department of Gastroenterology and Hepatology, The Second Affiliated \\ Hospital of Chongqing Medical University, Chongqing 400010, P.R. China
}

Received March 24, 2015; Accepted May 27, 2015

DOI: 10.3892/or.2015.4099

\begin{abstract}
MicroRNAs (miRNAs) are abnormally expressed in various types of cancer. miR-130a expression and function in gastric cancer has yet to be elucidated. The aim of the present study was to identify the miR-130a expression and function in gastric cancer. miR-130a expression was examined in gastric cancer cell lines and tissues by RT-qPCR. The diagnostic and prognostic significance of miR-130a in gastric cancer was analyzed by receiver-operating characteristic (ROC) curve and Kaplan-Meier analysis. miR130a expression was identified and the diagnostic significance in the serum of gastric cancer patients and healthy controls was analyzed using RT-qPCR and ROC curves, respectively. A target gene for miR-130a was identified using luciferase reporter assays, and gastric cancer tumorigenesis ability was examined by 3-(4,5-dimethylthazol2-yl)-2,5-diphenyltetrazolium bromide (MTT) and Transwell assays. The results showed that miR-130a was upregulated in gastric cancer. The low-miR-130a group had significantly improved overall survival compared to the high-miR-130a group. Furthermore, the expression of miR-130a in plasma in gastric cancer patients was upregulated and diagnostic value for gastric cancer of miR-130a is more effective than the tumor markers carcinoembryonic antigen (CEA) and CA-199. miR-130a directly targeted runt-related transcription factor 3 (RUNX3) and promoted gastric cancer tumorigenesis by targeting RUNX3. miR-130a may therefore be a useful marker for the diagnosis and prognosis of gastric cancer. Additionally, miR-130a was identified as an oncogene that promotes gastric cancer tumorigenesis by targeting RUNX3.
\end{abstract}

Correspondence to: Dr Yang Peng, Department of Geriatrics, Tongji Hospital, Tongji Medical College, Huazhong University of Science and Technology, 1095 Jiefang Road, Wuhan, Hubei 430030, P.R. China

E-mail:49074835@qq.com

Key words: miR-130a, gastric cancer, diagnosis, prognosis, RUNX3, tumorigenesis

\section{Introduction}

Gastric cancer is a common disease worldwide and the second most frequent cause of cancer-associated mortality, affecting approximately one million individuals annually (1). Of 880,000 people diagnosed with gastric cancer in 2000 , approximately 650,000 (74\%) succumbed to the disease (2). The genesis and progression of human gastric cancer is thought to be crucially influenced by genetic and epigenetic alterations, including the activation of oncogenes and the inactivation of tumor-suppressor genes (3). Oncogenes and tumor-suppressor genes have always been regulated by microRNAs (miRNAs).

miRNAs are non-coding RNA molecules $21-23$ nucleotides long that regulate gene expression at the post-transcriptional level (4-6). miRNA expression profiling analyses have revealed a global dysregulation of mature miRNA levels in primary human tumors compared to normal tissues $(7,8)$. miRNAs act as novel oncogenes or tumor-suppressor genes $(9,10)$ and it has been shown that alterations in microRNA expression correlate highly with the progression and prognosis of human tumors $(11,12)$. Furthermore, a number of differentially expressed miRNAs derived from circulation are used as potential biomarkers in various types of cancer, including liver, prostate, lymphoma and ovarian cancer (13-16). Thus, focusing on miRNAs in gastric cancer resulted in insight into the diagnosis and treatment of this disease.

An increasing body of evidence indicates miR-130a is differentially expressed in various tumors. miR-130a was overexpressed in adult T-cell leukemia (ATL), basal cell carcinoma and esophageal cancer tissue, but underexpressed in bladder and ovarian cancer, and glioblastoma (17-22). However, few studies have focused on miR-130a expression and its function in gastric cancer. Xu et al found that miR-130a directly inhibited the expression of tumor-suppressor gene runt-related transcription factor 3 (RUNX3) (23). RUNX3, a member of the family of transcription factors that contain the runt domain, is located at human chromosome 1p36 and was identified as a tumor-suppressor in breast, bladder and lung cancer (24-26). Previous findings showed that, RUNX3 was identified as a pivotal tumor-suppressor in gastric cancer (27), and a loss or substantial decrease in RUNX3 expression may 
be causally associated with gastric cancer, as it correlates with differentiation, lymph node metastasis and poor prognosis of this disease (28). Thus, miR-130a may act as an oncogene in gastric cancer by targeting RUNX3. Therefore, further systemic delineation of miR-130a expression and function in gastric cancer is needed.

\section{Materials and methods}

Human tissue specimens and cell lines. The present study utilized fresh tissues, including 41 human gastric cancer samples and 41 samples of adjacent normal mucosal tissues derived from 41 patients who underwent surgery at the Second Affiliated Hospital of Chongqing Medical University (Chongqing, China) between 2010 and 2011. The present study was conducted according to the 'Biomedical Research Involving Human Ethics Review (Tentative)' regulation of the Ministry of Health and the Declaration of Helsinki on Ethical Principles for Medical Research Involving Human Subjects. All the samples were obtained with the informed consent of the patients, and the experiments were approved by the Institutional Review Board of the Second Affiliated Hospital of Chongqing Medical University (Chongqing, China). All the participants provided written informed consent to participate in the present study.

The SGC-7901, HGC-27, AGS, MKN45 and N87 cell lines were obtained from the American Type Culture Collection (ATCC; Manassas, VA, USA), and the GES-1 cell line was purchased from the Type Culture Collection of the Chinese Academy of Sciences (Shanghai, China). The cell lines were cultured in RPMI-1640 (HyClone, Logan, UT, USA) supplemented with $10 \%$ fetal bovine serum (FBS) and were incubated at $37^{\circ} \mathrm{C}$ with $5 \% \mathrm{CO}_{2}$.

Serum collection. Whole blood $(2 \mathrm{ml})$ from the gastric cancer patients and healthy controls was collected in regular tubes and immediately processed to prevent contamination by cellular nucleic acids. Blood samples were centrifuged at 2,000 $\mathrm{rpm}$ for $10 \mathrm{~min}$ at room temperature, and then the upper supernatant, which was the serum sample, was transferred to new RNase-free collection tubes, respectively, and stored at $-80^{\circ} \mathrm{C}$ for further processing.

Detection of CEA and CA-199. Values for carcinoembryonic antigen (CEA) and carbohydrate antigen 199 (CA-199) levels in the serum of the gastric cancer patients and healthy controls were determined at the Clinical Laboratory of the Second Affiliated Hospital of Chongqing Medical University (Chongqing, China).

Primers, RNA isolation and miRNA detection. The primers for miR-338-3p and U6 were produced using the miScript Primer Assay kit (Qiagen, Dusseldorf, Germany). The sequences of the miRNAs used in the present study were as follows: miR-130a, CAGUGCAAUGUUAAAAGGGCAU; and U6, CGCAAGGA UGACACGCAAAUUCGUGAAGCGUUCCAUAUUUUU. The reverse primers were also used in the reverse transcription step. Total miRNA was extracted from the cultured cells, human tissue specimens and serum sample using RNAiso for small RNA (Takara Bio, Otsu, Japan) according to the manu- facturer's instructions. Poly(A) tails were added to miR-338 and U6 with the miRNA Reaction Buffer Mix, and then cDNA was produced from $5 \mathrm{ng}$ of total RNA using the miRNA PrimeScript RT Enzyme Mix (both from Takara Bio). RT-qPCR was performed in a CFX96 ${ }^{\mathrm{TM}}$ Real-Time PCR Detection System (Bio-Rad, Hercules, CA, USA) with SYBR ${ }^{\circledR}$ Premix Ex Taq ${ }^{\mathrm{TM}}$ II (Takara Bio). The PCR conditions used were $95^{\circ} \mathrm{C}$ for $30 \mathrm{sec}$, followed by 40 cycles of $95^{\circ} \mathrm{C}$ for $5 \mathrm{sec}$, and $60^{\circ} \mathrm{C}$ for $30 \mathrm{sec}$. The data were normalized against the U6 snRNA. After amplification, a melting curve analysis was performed to confirm the specificity of the products.

Expression levels of the miRNAs were calculated by cycle threshold $(\mathrm{Ct})$ values with SDS 2.0 software (Applied Biosystems, Foster City, CA, USA). The concentrations from serum, tissues or cell lines samples were normalized using the $2^{-\Delta \Delta \mathrm{Ct}}$ method relative to U6 small nuclear RNA (RNU6B). The value of $\Delta \mathrm{Ct}$ was calculated by subtracting the $\mathrm{Ct}$ values of RNU6B from the $\mathrm{Ct}$ values of the miRNAs of interest in the present study. The values of $\Delta \Delta \mathrm{Ct}$ were then calculated by subtracting the $\Delta \mathrm{Ct}$ of the control samples from the $\Delta \mathrm{Ct}$ of the cancer samples. The change in gene expression was calculated using the equation $2^{-\Delta \Delta \mathrm{C} t}$.

Oligonucleotide transfection. miR-130a mimics, inhibitor and cont-miR were produced by Sangon Biotechnology (Sangon, Shanghai, China), and co-transfections were performed with Lipofectamine 2000 (Invitrogen-Life Technologies, Carlsbad, CA, USA). Twenty-four hours after transfection, the cells were plated for hte proliferation, migration and invasion assays. The cells were collected for RNA and protein analyses $48 \mathrm{~h}$ after transfection.

pcDNA expression plasmids and plasmid transfection. The ORF sequences of RUNX3 were amplified from genomic DNA isolated from the AGS cell line and were then subcloned into the GV230 vector (GeneChem Corporation, Shanghai, China). The plasmid was transfected into AGS cells using Lipofectamine 2000. Twenty-four hours after transfection, the cells were used for a rescue experiment.

Luciferase reporter assay. A Psicheck ${ }^{\mathrm{TM}}-2$ Dual-Luciferase miRNA target expression vector was used for the 3'UTR luciferase assays (Sangon Biotechnology). The target gene of miRNA-130a was selected based on the online microRNA target database, http://www.microrna.org/microrna/home.do. The primer sequences used for the wild-type 3'UTR of RUNX3 were: forward, 5'-CCGCCCTGGTGGACTCCT-3' and reverse, 5'-CCTTCCACACATCTCAGAGTTATAT-3'. Since there was one binding site in the RUNX3 3'UTR, primer sequences were designed for the mutant $3^{\prime} \mathrm{UTR}$ as follows: forward, 5'-GTGGAAACTGTGGCGCGCCATCGTTTGC TTGGTGTTTG-3' and reverse, 5'-GCAAACGATAGTGCA AAGCAGTTTCCACCCAGCTCCAT-3'. For the luciferase assay, Lipofectamine 2000 was used to co-transfect MKN45 cells with the miR-130a mimics and Psicheck ${ }^{\mathrm{TM}}-2$ DualLuciferase miRNA target expression vectors containing wildtype or mutant target sequences. The Dual-Luciferase Assay (Promega, Madison, WI, USA) was used to measure the firefly luciferase activity $18 \mathrm{~h}$ after transfection, and the results were normalized against the Renilla luciferase. Each reporter 
A

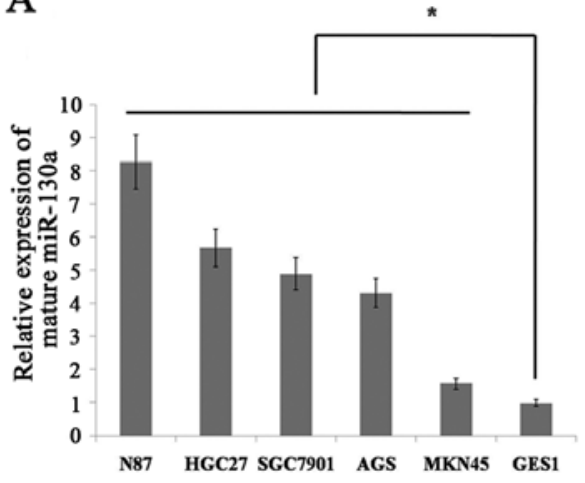

B

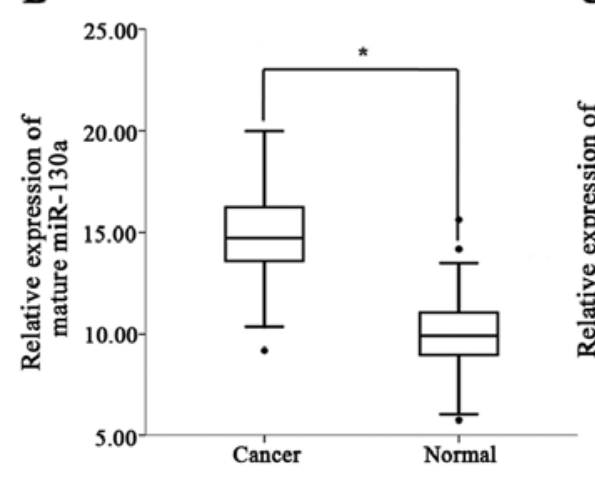

$\mathrm{C}$

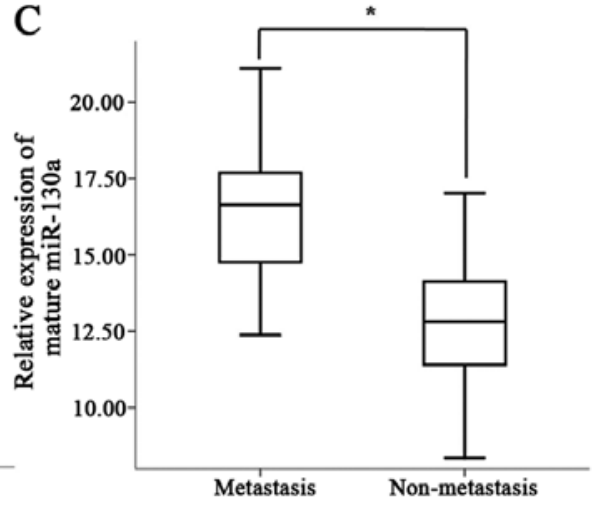

Figure 1. miR-130a is upregulated in gastric cancer tissues and cell lines. (A) miR-130a expression was detected in gastric cancer cell lines (SGC7901, HGC27, AGS, MKN45 and N87) and a normal gastric mucosa cell line, GES1. Data are shown as the mean \pm SD $(n=3)$ in the cell lines, ${ }^{*} \mathrm{p}=0.021$. (B) The expression level of mature miR-130a in gastric cancer $(\mathrm{n}=41)$ or adjacent normal mucosal tissues $(\mathrm{n}=41)$ was determined by RT-qPCR analysis. Data are shown separately in human samples, ${ }^{*} \mathrm{p}=0.018$. (C) Mature miR-130a expression levels in metastatic $(\mathrm{n}=25)$ and non-metastatic $(\mathrm{n}=16)$ gastric cancers. Data are shown separately in human samples, ${ }^{*} \mathrm{p}=0.009$.

plasmid was transfected at least three times (on different days), and each sample was assayed in triplicate.

Cell viability assays. The transfected cells were seeded in 96-well plates at a density of $1 \times 10^{4}$ cells/well. A 3-(4,5-dimethylthazol-2-yl)-2,5-diphenyltetrazolium bromide (MTT) solution (20 $\mathrm{ml}$ of $5 \mathrm{mg} / \mathrm{ml} \mathrm{MTT}$ ) was added to the cultures (for a total volume of $250 \mu \mathrm{l}$ ) and incubated for $4 \mathrm{~h}$ at $37^{\circ} \mathrm{C}$. Following the removal of the culture medium, the remaining crystals were dissolved in dimethylsulfoxide (DMSO), and the absorbance at $570 \mathrm{~nm}$ was measured.

Migration and invasion assays. For the Transwell migration assays, $1 \times 10^{4}$ cells were plated in the top chamber with a non-coated membrane (24-well insert; 8 -mm pore size; BD Biosciences, Franklin Lakes, NJ, USA). For the invasion assays, $2 \times 10^{5}$ cells were plated in the top chamber with a Matrigel-coated membrane (24-well insert; $8 \mathrm{~mm}$ pore size; BD Biosciences). For the two assays, the cells were plated in a serum-free medium, and medium supplemented with $10 \%$ serum was used as a chemoattractant in the lower chamber. The cells were incubated for $16 \mathrm{~h}$ at $37^{\circ} \mathrm{C}$ and $5 \% \mathrm{CO}_{2}$ in a tissue culture incubator. After $16 \mathrm{~h}$, the non-migrated/noninvading cells were removed from the upper sides of the Transwell membrane filter inserts using cotton-tipped swabs. The migrated/invaded cells on the lower sides of the inserts were stained with Giemsa, and the cells were counted.

Antibodies and immunoblotting. Antibodies against RUNX3 and Smad4 were purchased from Abcam (Cambridge, UK). Antibody against GAPDH was purchased from Santa Cruz Biotechnology, Inc. (Santa Cruz, CA, USA). HRP-conjugated goat anti-rabbit IgG was purchased from Santa Cruz Biotechnology, Inc.. The total protein was extracted from the transfected cells and gastric cancer tissues using RIPA lysis buffer (Beyotime, China) according to the manufacturer's instructions. After the whole-cell protein extracts were quantified using the BCA protein assay, equivalent amounts of cell lysates were resolved by $10 \%$ SDS polyacrylamide gel electrophoresis, and were transferred onto a polyvinylidene fluoride membrane, which was then blocked in 5\% non-fat milk in TBST for $1 \mathrm{~h}$ at $4^{\circ} \mathrm{C}$. The blots were then incubated with primary antibodies. After incubation with HRP-conjugated secondary antibodies, the protein bands were visualized using an enhanced chemiluminescence reagent (Millipore, Billerica, MA, USA). The following antibody dilutions were used: anti-RUNX and anti-Smad4, 1:1,500; and HRP-conjugated IgG, 1:7,000.

Statistical analysis. SPSS 17.0 software was used for the statistical analysis. The data are presented as the means \pm standard deviation (SD). Group comparisons were performed using the Student's t-test. The relationships between miR-130a expression in the serum of gastric cancer patients and healthy control were analyzed using the non-parametric Mann-Whitney $\mathrm{U}$ test. Receiver-operating characteristic (ROC) curves and the area under the ROC curve (AUC) were used to assess the feasibility of serum and tissue miRNA as a diagnostic tool for detecting gastric cancer. For disease progression, the Kaplan-Meier (log-rank test) analysis was performed. The Spearman's rank test was used to evaluate the relationships among the relative expression levels of miR-130a and RUNX3 in gastric cancer tissues. The relationships, in the AGS cell line with the forced expression of miR-130a or cont-miR and with or without RUNX3 restoration, were analyzed using the one-way ANOVA Dunnett's test. Differences were considered to indicate a statistically significant result when $\mathrm{P}<0.05$.

\section{Results}

miR-130a is upregulated in gastric cancer tissues and cell lines. To investigate the miR-130a expression levels in human gastric cancer cell lines, we monitored its expression in several cancer cell lines (SGC7901, HGC27, AGS, MKN45 and N87) and a normal gastric mucosal cell line (GES1). We found that miR-130a expression was increased in gastric cancer cell lines compared to the normal gastric mucosal cells (Fig. 1A). To further examine the role of miR-130a in human gastric cancer development, we detected the levels of its expression in 41 cases of human gastric cancer and 41 cases adjacent normal 
Table I. Clinicopathological characteristics of the patient cohort.

\begin{tabular}{lc}
\hline Characteristics & Total cases $(\mathrm{n}=41)$ \\
\hline Age (years) & $33-88$ \\
Range & 54 \\
Mean & 57 \\
Median & \\
Pathological T $(\%)$ & $12(29.2)$ \\
$\mathrm{PT}_{-} \mathrm{T}_{\text {is }}$ & $9(22.0)$ \\
$\mathrm{PT}_{1}$ & $6(14.6)$ \\
$\mathrm{PT}_{2}$ & $8(19.5)$ \\
$\mathrm{PT}_{3}$ & $6(14.7)$ \\
$\mathrm{PT}_{4}$ & \\
Lymph node metastases $(\%)_{\mathrm{PN}_{0}}$ & $16(39.0)$ \\
$\mathrm{PN}_{1-3}$ & $25(61.0)$ \\
Dead/alive $(\%)$ & \\
Dead & $25(61.0)$ \\
Alive & $16(39.0)$ \\
\hline
\end{tabular}

mucosa tissues. The RT-qPCR analysis revealed that, the levels of miR-130a expression were significantly increased in tumor tissues compared to that of the adjacent normal mucosal tissues (Fig. 1B). To determine whether miR-130a expression was associated with gastric cancer metastasis, we examined the miR-130a expression levels in 41 archived primary gastric tumors. These tumors were divided into two groups: in one group tumors were resected from 25 patients with lymph node metastasis, while in the second group the tumors were resected from 16 patients without metastasis. The RT-qPCR analysis revealed that, the miR-130a expression levels were significantly higher in the patients with metastasis than in those without metastasis.

Diagnostic and prognostic significance of miR-130a in gastric cancer. Clinical information for the 41 patients is shown in Table I. Receiver-operating characteristic (ROC) curve analyses were performed to evaluate the ability of miR-130a expression to discriminate between normal and tumor cases using tissue samples. According to ROC curve, miR-130a had the best sensitivity and specificity when the miR-130a Ct value was 11.13 ( $\mathrm{YI}=0.634)$, and an $\mathrm{AUC}$ of $0.905(\mathrm{P}=0.0001$; 95\% CI, 0.841-0.969) (Fig. 2A) was obtained, suggesting that miR-130a expression discriminated between malignant and non-malignant samples and may therefore be used as a diagnostic marker for gastric cancer. To determine whether the levels of miR-130a in tumor tissues correlate with the survival of the gastric cancer patients, the patients were divided into low-miR-130a (expression $\mathrm{Ct}$ value $<11.13$ ) and high-miR-130a (expression $\mathrm{Ct}$ value $\geq 11.13$ ) groups and a Kaplan-Meier survival analysis was performed. The results of the Kaplan-Meier analysis revealed that the low-miR-130a group had a significantly improved overall survival compared to the high-miR-130a group (Fig. 2B).
Table II. Area under the ROC curve analysis of CEA, CA-199 and miR-130a expression in the serum samples of the gastric cancer patients and controls.

\begin{tabular}{lcccccc}
\hline & & & & \multicolumn{2}{c}{$\begin{array}{c}\text { Asymptotic } \\
95 \%\end{array}$} \\
\cline { 5 - 7 } & & & & CI \\
\cline { 5 - 7 } Variables & Area & SE $^{\mathrm{a}}$ & $\begin{array}{c}\text { Asymptotic } \\
\text { significance }\end{array}$ & $\begin{array}{l}\text { Lower } \\
\text { bound }\end{array}$ & $\begin{array}{c}\text { Upper } \\
\text { bound }\end{array}$ \\
\hline CA-199 & 0.750 & 0.054 & 0.000 & 0.644 & 0.855 \\
CEA & 0.749 & 0.053 & 0.000 & 0.644 & 0.853 \\
miR-130a & 0.870 & 0.040 & 0.000 & 0.792 & 0.948 \\
\hline
\end{tabular}

Statistics may be biased. ${ }^{\mathrm{a} U n d e r}$ the non-parametric assumption; ${ }^{\mathrm{b}}$ null hypothesis, true area, 0.5 . SE, standard error. CI, confidence interval; ROC, receiver-operating characteristic; CEA, carcinoembryonic antigen.

miRl30a expression and diagnostic significance in the serum of gastric cancer patients and healthy controls. Clinicopathological characteristics for the gastric cancer patients are shown in Table I. miR-130a expression was detected in the serum of gastric cancer patients and healthy controls. We also found that the expression of miR-130a in plasma in 41 gastric cancer patients was significantly higher than that from the healthy controls (Fig. 3A). To further understand the significance of the diagnostic value between miR-130a and traditional tumor markers CEA and CA-199, a ROC analysis of CEA, CA-199 and miR-130a was performed. Regarding the area under the ROC curve, miR-130a had the highest value at 0.870 , while values for CEA and CA-199 were at 0.749 and 0.750, respectively (Fig. 3B and Table II). Our findings showed that the diagnostic value of miR-130a was more effective than that for tumor markers CEA and CA-199 in the serum of gastric cancer patients and healthy controls.

miR-130a directly targets RUNX3. We predicted 26 targets for miR-130a using the following prediction tools: miRanda (http://www.microrna.org), TargetScan (http://www. targetscan.org/) and miRDB (mirdb.org/miRDB/). In those 26 targets, we found some genes associated with tumors, such as RUNX3, MET, Smad4 and KLF4 (Table III). Recent findings showed that, RUNX3 and Smad4, which function as tumor-suppressor genes, are associated with gastric cancer (29-31). To determine whether miR-130a targets RUNX3 and Smad4, we examined RUNX3 and Smad4 expression in the AGS gastric cell line following transfection with miR-130a inhibitor. Compared with the expression of cont-miR, miR130a was significantly downregulated in the AGS cell line following transfection with the miR-130a inhibitor (Fig. 4A), and we found that RUNX3 expression was significantly increased in miR-130a-low-expressing gastric cancer cell lines, although Smad4 expression was not increased (Fig. 4B). Our findings showed that RUNX3 may be the target gene for miR130a. To confirm that miR-130a directly targeted RUNX3, we performed luciferase reporter assays to examine whether miR-130a interacts directly with RUNX3. We found that the co-transfection of miR-130a and the wild-type RUNX3 3'UTR 
Table III. miR-130a predicted targets.

Total target genes

Target genes associated with tumors

CTSA, STK38L, ZFPM2, BMPR2, ACVR1, LDLR,

DNM2, ATG2B, DICER1, PPARG, KLF4, HOXA10,

HoxA5, MAFB, ESR 1, IFITM1, RUNX3,

CSF1, MEOX2, APP, ATXN1, TAC1, HoxA5, MAFB,

MET, RAB5A, Smad4, Smad5,

ESR1, IFITM1, RUNX3, MET, RAB5A, Smad4, Smad5

ATG2B, KLF4, HOXA10

RUNX3, runt-related transcription factor 3 .

A

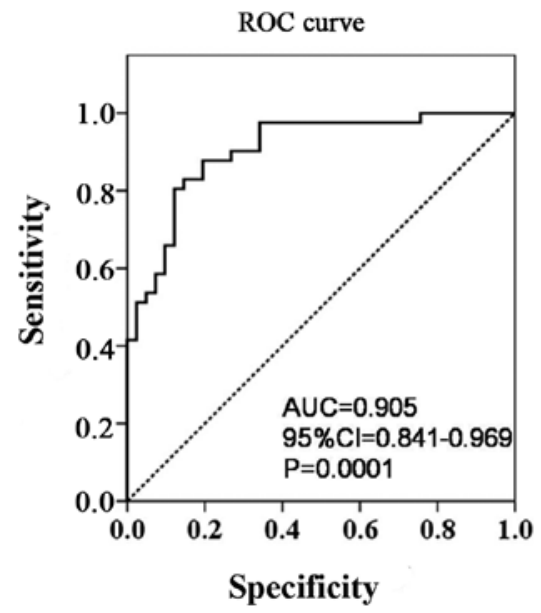

B $\quad$ Low miR-130a

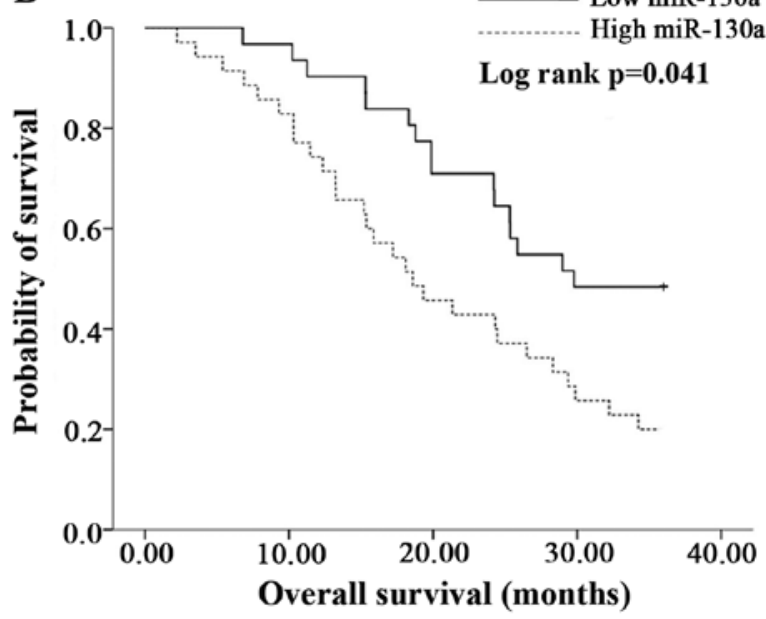

Figure 2. Diagnostic and prognostic significance of miR-130a is shown in gastric cancer. (A) ROC curve analysis showing performance of miR-130a expression to discriminate between malignant and non-malignant tissue samples. (B) Kaplan-Meier analysis for overall survival based on miR-130a expression. ROC, receiver-operating characteristic.
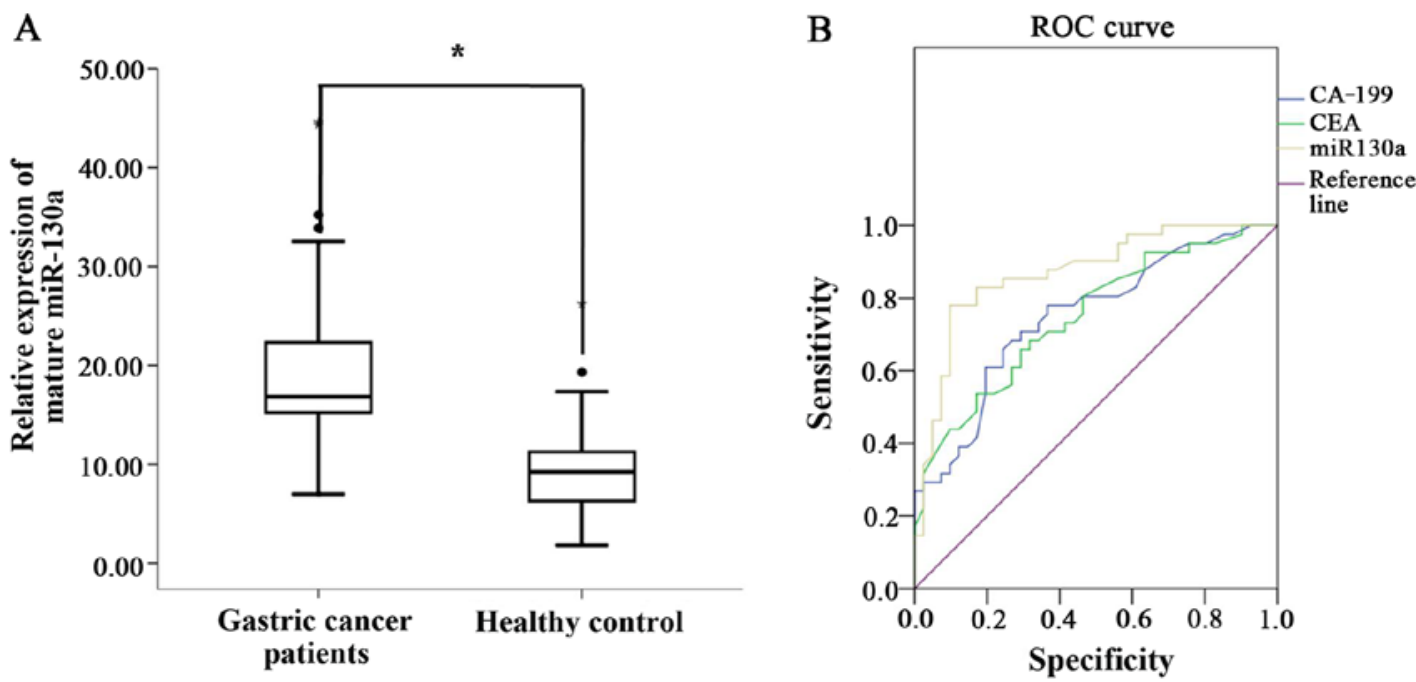

Figure 3. miR130a expression and diagnostic significance is shown in the serum of gastric cancer patients and healthy controls. (A) The expression level of mature miR-130a in gastric cancer patients $(n=41)$ or healthy patients $(n=41)$ was determined by RT-qPCR analysis. Data are shown separately in human serum samples, ${ }^{*} \mathrm{p}=0.0041$. (B) ROC analysis curve of CEA, CA-199 and miR-130a expression in the serum of the gastric cancer patients and healthy controls. ROC, receiver-operating characteristic; CEA, carcinoembryonic antigen.

caused a significant decrease in luciferase expression when compared with the controls. However, the co-transfection of miR-130a and the mutant RUNX3 3'UTR did not cause a decrease in luciferase expression (Fig. 4C). The RUNX3 
A

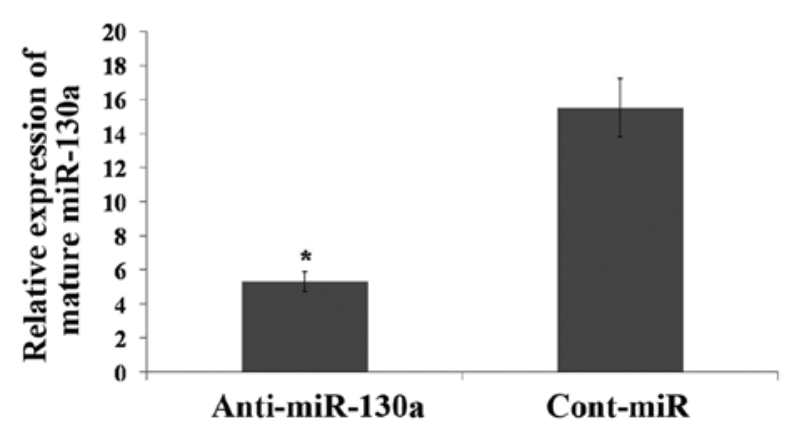

C

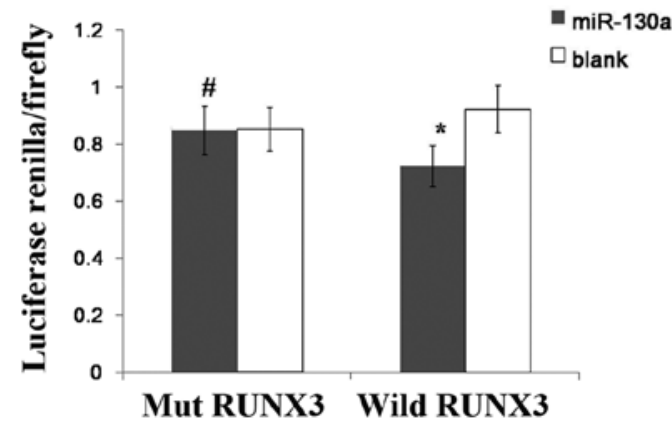

B

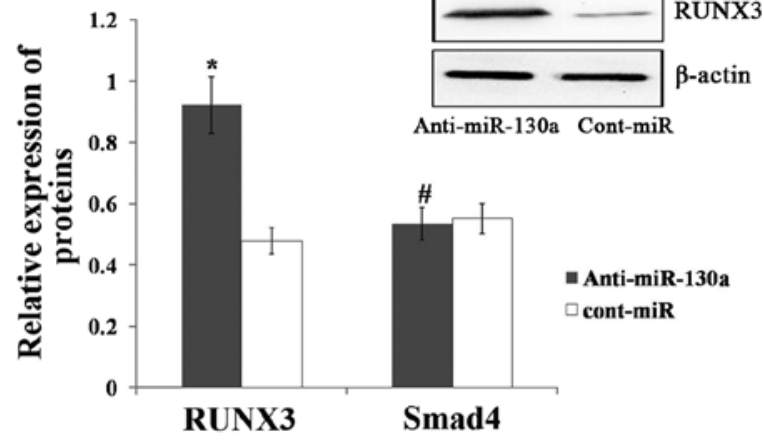

D

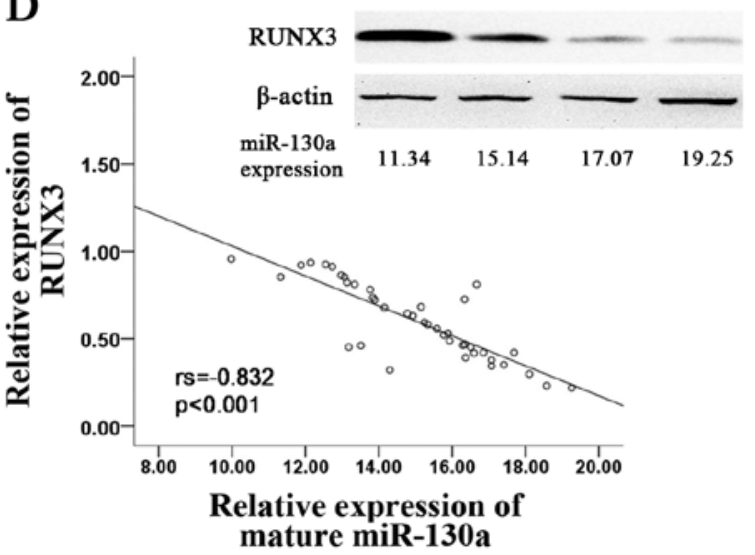

Figure 4. miR-130a directly targets RUNX3. (A) miR-130a expression was detected in the AGS gastric cell line after transfecting anti-miR-130a and cont-miR. "P=0.0018 compared to the controls. (B) Smad4 and RUNX3 expression was examined by western blotting in the AGS gastric cell line following transfection with anti-miR-130a and cont-miR. ${ }^{*} \mathrm{P}=0.031$ and ${ }^{\#} \mathrm{p}=0.322$ compared to the controls. (C) The relative luciferase activity was analyzed after the reporter plasmids were co-transfected with miR-130a mimics or control mimics into the AGS cell lines. ${ }^{*} \mathrm{P}=0.019$ and ${ }^{\#} \mathrm{p}=0.462$ compared to the controls. (D) RUNX3 protein expression was examined by western blotting in gastric cancer tissues and the correlation between miR-130a and RUNX3 protein expression is shown. RUNX3, runt-related transcription factor 3.

expression was also detected in 41 gastric cancer tissues by western blotting, and we found that the expression of miR-130a was inversely correlated with that of RUNX3 (Fig. 4D). These results suggested that miR-130a directly targets RUNX3.

miR-130a promotes gastric cancer cell migration, invasion and proliferation by targeting RUNX3. To determine the functional significance of miR-130a in gastric cancer, we transfected the AGS and MKN45 gastric cancer cell lines with miR-130a mimics. We found that the cells with a forced expression of miR-130a significantly increased proliferation compared with the cells with a forced expression of cont-miR (Fig. 5A). Transwell migration and Matrigel invasion assays demonstrated that miR-130a significantly increased the migration and invasion of AGS and MKN45 cells (Fig. 5B). To confirm whether miR-130a promotes gastric cancer migration, invasion and proliferation by targeting RUNX3, we forced the expression of miR-130a in AGS and MKN45 cell lines along with a construct containing the RUNX3 coding sequence but lacking the 3'UTR of the RUNX3 mRNA. As a result, this construct yielded a RUNX3 mRNA that was resistant to miR130a. The restoration of RUNX3 expression was confirmed through an immunoblot analysis (Fig. 5C). We found that the gastric cancer cell migration, invasion and proliferation were completely restored in the AGS cell line with a forced
miR-130a expression and RUNX3 restoration (Fig. 5D). Therefore, miR-130a regulated gastric cancer cell migration, invasion and proliferation by targeting RUNX3.

\section{Discussion}

In the present study, we did examined miR-130a expression in gastric cancer cell lines and detected miR-130a expression in gastric cancer tissues, and found that miR-130a expression was significantly upregulated in gastric cancer cell lines and tissues. Furthermore, miR-130a expression was significantly higher in the patients with metastasis than in the patients without metastasis. The results show that miR-130a as an oncogene was overexpressed in gastric cancer and was associated with metastasis. miRNAs possess several features that make them attractive candidates as new prognostic biomarkers and powerful tools for the early diagnosis of cancer $(32,33)$. ROC and Kaplan-Meier analysis were than performed to determine the miR-130a diagnostic and prognostic potential in gastric cancer. Our findings show that miR-130a can be used as a diagnostic marker for gastric cancer and low levels of miR-130a were significantly associated with an extended overall survival of gastric cancer patients. Thus, miR-130a serves as a molecular diagnostic and prognostic marker for gastric cancer patients. 

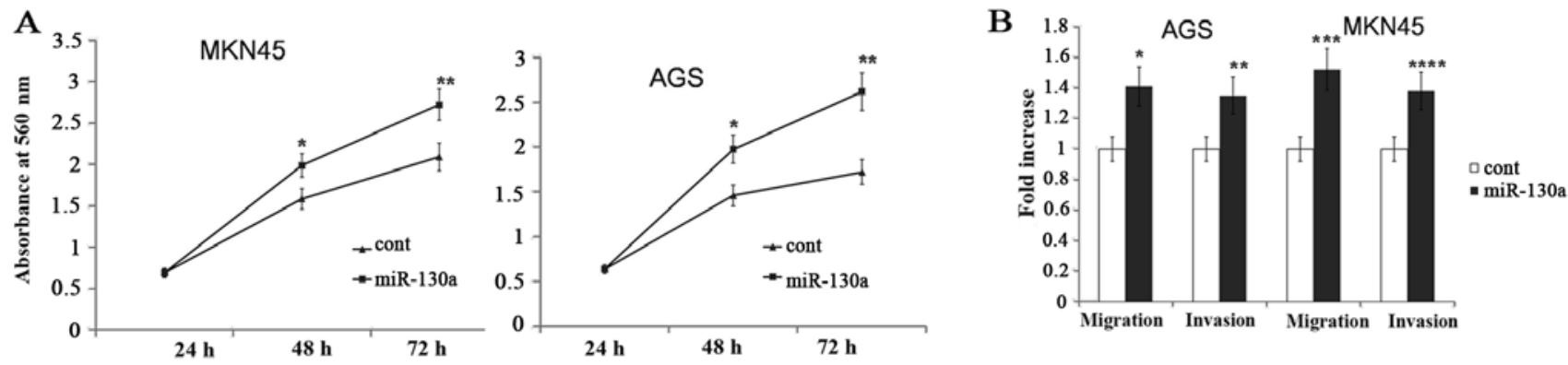

C

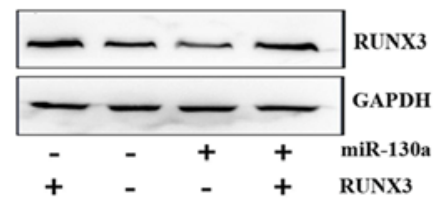

D

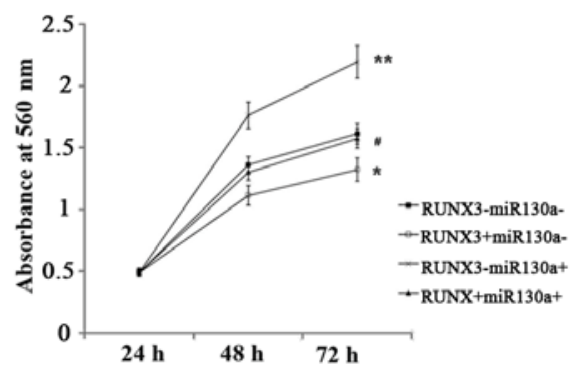

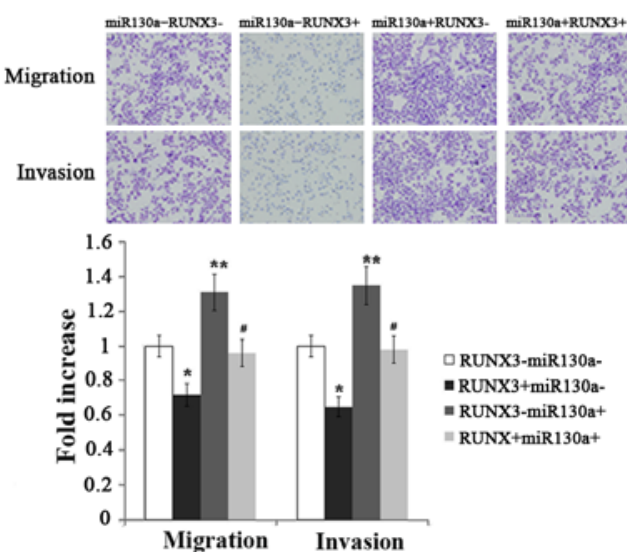

Figure 5. miR-130a promotes gastric cancer migration, invasion and proliferation by targeting RUNX3. (A) Cell proliferation is shown after transfection with miR-130a mimics or cont-miR in the AGS and MKN45 gastric cancer cell lines. ${ }^{*} \mathrm{P}=0.031$ and ${ }^{* *} \mathrm{p}=0.022$ for the AGS cell line and ${ }^{*} \mathrm{p}=0.031$ and ${ }^{* *} \mathrm{p}=0.015$ for the MKN45 cell line compared to the controls. (B) The migration and invasion of AGS and MKN45 cell lines were exhibited following transfection with miR-130a mimics or cont-miR: ${ }^{*} \mathrm{p}=0.0018,{ }^{* *} \mathrm{p}=0.0032,{ }^{* * *} \mathrm{p}=0.0011$ and ${ }^{* * * *} \mathrm{p}=0.012$ compared to the controls. (C) Immunoblot analysis of RUNX3 expression in AGS cells transfected with miR-130a mimics or cont-miR with or without RUNX3 restoration. (D) The tumorigenic qualities of AGS cells were detected following transfection with miR-130a or cont-miR and with or without RUNX3 restoration. Cell proliferation is shown in the left panel: " $\mathrm{p}=0.0128$, ${ }^{* * *} \mathrm{p}=0.0022$ and ${ }^{\#} \mathrm{p}=0.312$. Gastric cancer migration and invasion are shown in right panel: Migration ${ }^{*} \mathrm{p}=0.0328,{ }^{* *} \mathrm{p}=0.0122$, and ${ }^{*} \mathrm{p}=0.215$; and invasion ${ }^{*} \mathrm{p}=0.0328$, ** $\mathrm{p}=0.0125$ and ${ }^{\#} \mathrm{p}=0.415$ compared to controls.

Screening for early gastric cancer potentially reduces mortality of the disease (34-36). Since the currently known tumor markers have the limitation of low sensitivity and specificity (37), diagnosis of gastric cancer is performed using a gastroscopic biopsy sample and histology specified by WHO criteria. Although gastroscopic screening for gastric cancer is currently the most reliable screening tool, it is expensive and unsuitable as a first-line examination due to the invasive nature. Thus, identification of novel non-invasive biomarkers for tumor detection is imperative. Previous findings have shown that some miRNAs have potential diagnostic value in different types of cancer $(19,38,39)$. In the present study, we found that miR-130a expression in serum was overexpressed in gastric cancer patients than healthy controls. Compared with the diagnostic value of CEA, CA-199 and miR-130a individually, miR-130a had the highest value at 0.870 on the area under the ROC curve. miR-130a is sufficiently reliable for the diagnosis of gastric cancer. Thus, miR-130a acts as a potential circulating diagnostic biomarker for gastric cancer.

Some miRNAs inhibit the expression of tumor-suppressor genes in normal tissues, elucidating the reason for miRNAs acting as oncogenes. Chu et al found that miR-590-5p acts as an oncogene by targeting the CHL1 gene and promotes cervical cancer proliferation (40). Ma et al also found that miR-34a targets GAS1 to promote cell proliferation and inhibit apoptosis in papillary thyroid carcinoma (41). To predict target genes of miR-130a, we screened 26 targets for miR-130a by prediction tools, and found that 12 target genes were associated with tumors. We focused on RUNX3 and Smad4 since it has been previously shown that RUNX3 and Smad4 as tumor suppressor genes are associated with gastric cancer. RUNX3 has been thought to be the target gene of miR$130 \mathrm{a}$ in other tissues. It has been shown that miR-130a directly inhibited the expression of the tumor-suppressor gene RUNX3, which resulted in the activation of $W n t / \beta$-catenin signaling and increased drug resistance (23). miR-130a also contributes to endothelial progenitor cell dysfunction by targeting RUNX3 (42).

In the present study, Smad4 was excluded since the inhibited expression of miR-130a did not increase Smad4 expression, although RUNX3 expression was significantly increased following transfection with anti-miR-130a. miR-130a expression was inversely correlated with that of RUNX3 in gastric cancer tissues. The result shows that miR-130a attenuates RUNX3 expression in gastric cancer tissues. Thus, RUNX3 may be a target gene of miR-130a. Luciferase reporter assays were performed to confirm miR-130a directly targets RUNX3, and we found that miR-130a directly interacts with RUNX3 by binding to RUNX3 3'UTR. Thus, miR-130a can directly target tumor-suppressor gene $R U N X 3$. Furthermore we found that miR-130a significantly increases gastric cancer cell migration, invasion and proliferation, which was completely restored after 
RUNX3 restoration. The results show that miR-130 promotes gastric cancer tumorigenic abilities by targeting RUNX3, and it elucidates the reason for miR-130a acting as an oncogene in gastric cancer.

In conclusion, the results show that miR-130a expression was increased in gastric cancer and was associated with the overall survival of gastric cancer. miR-130a can be a potential circulating diagnostic biomarker for gastric cancer. Thus, miR130a may be used as a biomarker for gastric cancer diagnosis and prognosis. Furthermore, miR-130a acts as an oncogene that promotes gastric cancer tumorigenesis by targeting the tumor-suppressor gene $R U N X 3$. Thus, future studies on the anticancer mechanisms of miR-130a may contribute to the development of new therapeutic strategies for gastric cancer.

\section{Acknowledgements}

We thank Dr Xiao-Qiu Xiao for his assistance with the RT-qPCR analysis.

\section{References}

1. Hartgrink HH, Jansen EP, van Grieken NC and van de Velde CJ: Gastric cancer. Lancet 374: 477-490, 2009.

2. Kamangar F, Dores GM and Anderson WF: Patterns of cancer incidence, mortality, and prevalence across five continents: Defining priorities to reduce cancer disparities in different geographic regions of the world. J Clin Oncol 24: 2137-2150, 2006.

3. Wu WK, Lee CW, Cho CH, Fan D, Wu K, Yu J and Sung JJ: MicroRNA dysregulation in gastric cancer: A new player enters the game. Oncogene 29: 5761-5771, 2010.

4. Rana TM: Illuminating the silence: Understanding the structure and function of small RNAs. Nat Rev Mol Cell Biol 8: 23-36, 2007.

5. Valencia-Sanchez MA, Liu J, Hannon GJ and Parker R: Control of translation and mRNA degradation by miRNAs and siRNAs. Genes Dev 20: 515-524, 2006.

6. Pillai RS, Bhattacharyya SN and Filipowicz W: Repression of protein synthesis by miRNAs: How many mechanisms? Trends Cell Biol 17: 118-126, 2007.

7. Lu J, Getz G, Miska EA, Alvarez-Saavedra E, Lamb J, Peck D, Sweet-Cordero A, Ebert BL, Mak RH, Ferrando AA, et al: MicroRNA expression profiles classify human cancers. Nature 435: 834-838, 2005

8. Thomson JM, Newman M, Parker JS, Morin-Kensicki EM, Wright $\mathrm{T}$ and Hammond SM: Extensive post-transcriptional regulation of microRNAs and its implications for cancer. Genes Dev 20: 2202-2207, 2006.

9. Esquela-Kerscher A and Slack FJ: Oncomirs - microRNAs with a role in cancer. Nat Rev Cancer 6: 259-269, 2006.

10. Calin GA and Croce CM: MicroRNA signatures in human cancers. Nat Rev Cancer 6: 857-866, 2006.

11. Schetter AJ, Leung SY, Sohn JJ, Zanetti KA, Bowman ED, Yanaihara N, Yuen ST, Chan TL, Kwong DL, Au GK, et al: MicroRNA expression profiles associated with prognosis and therapeutic outcome in colon adenocarcinoma. JAMA 299: 425-436, 2008.

12. Garzon R, Volinia S, Liu CG, Fernandez-Cymering C, Palumbo T, Pichiorri F, Fabbri M, Coombes K, Alder H, Nakamura T, et al: MicroRNA signatures associated with cytogenetics and prognosis in acute myeloid leukemia. Blood 111: 3183-3189, 2008.

13. Xu J, Wu C, Che X, Wang L, Yu D, Zhang T, Huang L, Li H, Tan W, Wang C, et al: Circulating microRNAs, miR-21, miR-122, and miR-223, in patients with hepatocellular carcinoma or chronic hepatitis. Mol Carcinog 50: 136-142, 2011.

14. Yaman Agaoglu F, Kovancilar M, Dizdar Y, Darendeliler E, Holdenrieder S, Dalay N and Gezer U: Investigation of miR-21, miR-141, and miR-221 in blood circulation of patients with prostate cancer. Tumour Biol 32: 583-588, 2011.
15. Lawrie CH, Gal S, Dunlop HM, Pushkaran B, Liggins AP, Pulford K, Banham AH, Pezzella F, Boultwood J, Wainscoat JS, et al: Detection of elevated levels of tumour-associated microRNAs in serum of patients with diffuse large B-cell lymphoma. Br J Haematol 141: 672-675, 2008.

16. Resnick KE, Alder H, Hagan JP, Richardson DL, Croce CM and Cohn DE: The detection of differentially expressed microRNAs from the serum of ovarian cancer patients using a novel real-time PCR platform. Gynecol Oncol 112: 55-59, 2009.

17. Qiu S, Lin S, Hu D, Feng Y, Tan Y and Peng Y: Interactions of $\mathrm{miR}-323 / \mathrm{miR}-326 / \mathrm{miR}-329$ and $\mathrm{miR}-130 \mathrm{a} / \mathrm{miR}-155 / \mathrm{miR}-210$ as prognostic indicators for clinical outcome of glioblastoma patients. J Transl Med 11: 10, 2013.

18. Ratert N, Meyer HA, Jung M, Lioudmer P, Mollenkopf HJ, Wagner I, Miller K, Kilic E, Erbersdobler A, Weikert S, et al: miRNA profiling identifies candidate miRNAs for bladder cancer diagnosis and clinical outcome. J Mol Diagn 15: 695-705, 2013.

19. Ishihara K, Sasaki D, Tsuruda K, Inokuchi N, Nagai K, Hasegawa H, Yanagihara K and Kamihira S: Impact of miR-155 and miR-126 as novel biomarkers on the assessment of disease progression and prognosis in adult T-cell leukemia. Cancer Epidemiol 36: 560-565, 2012.

20. Sand M, Skrygan M, Sand D, Georgas D, Hahn SA, Gambichler T, Altmeyer P and Bechara FG: Expression of microRNAs in basal cell carcinoma. Br J Dermatol 167: 847-855, 2012.

21. Liu SG, Qin XG, Zhao BS, Qi B, Yao WJ, Wang TY, Li HC and Wu XN: Differential expression of miRNAs in esophageal cancer tissue. Oncol Lett 5: 1639-1642, 2013.

22. Zhang X, Huang L, Zhao Y and Tan W: Downregulation of miR-130a contributes to cisplatin resistance in ovarian cancer cells by targeting X-linked inhibitor of apoptosis (XIAP) directly. Acta Biochim Biophys Sin 45: 995-1001, 2013.

23. Xu N, Shen C, Luo Y, Xia L, Xue F, Xia Q and Zhang J: Upregulated miR-130a increases drug resistance by regulating RUNX3 and Wnt signaling in cisplatin-treated HCC cell. Biochem Biophys Res Commun 425: 468-472, 2012.

24. Huang B, Qu Z, Ong CW, Tsang YH, Xiao G, Shapiro D, SaltoTellez M, Ito K, Ito Y and Chen LF: RUNX3 acts as a tumor suppressor in breast cancer by targeting estrogen receptor $\alpha$. Oncogene 31: 527-534, 2012.

25. Kim WJ, Kim EJ, Jeong P, Quan C, Kim J, Li QL, Yang JO, Ito Y and Bae SC: RUNX3 inactivation by point mutations and aberrant DNA methylation in bladder tumors. Cancer Res 65: 9347-9354, 2005.

26. Araki K, Osaki M, Nagahama Y, Hiramatsu T, Nakamura H, Ohgi $\mathrm{S}$ and Ito H: Expression of RUNX3 protein in human lung adenocarcinoma: Implications for tumor progression and prognosis. Cancer Sci 96: 227-231, 2005.

27. Li QL, Ito K, Sakakura C, Fukamachi H, Inoue K, Chi XZ, Lee KY, Nomura S, Lee CW, Han SB, et al: Causal relationship between the loss of RUNX3 expression and gastric cancer. Cell 109: 113-124, 2002.

28. Hsu PI, Hsieh HL, Lee J, Lin LF, Chen HC, Lu PJ and Hsiao M: Loss of RUNX3 expression correlates with differentiation, nodal metastasis, and poor prognosis of gastric cancer. Ann Surg Oncol 16: 1686-1694, 2009.

29. Kundu J, Wahab SM, Kundu JK, Choi YL, Erkin OC, Lee HS, Park SG and Shin YK: Tob1 induces apoptosis and inhibits proliferation, migration and invasion of gastric cancer cells by activating Smad 4 and inhibiting $\beta$-catenin signaling. Int $J$ Oncol 41: 839-848, 2012.

30. Fukamachi H: Runx 3 controls growth and differentiation of gastric epithelial cells in mammals. Dev Growth Differ 48: 1-13, 2006.

31. Cheng HC, Liu YP, Shan YS, Huang CY, Lin FC, Lin LC, Lee L, Tsai CH, Hsiao M and Lu PJ: Loss of RUNX3 increases osteopontin expression and promotes cell migration in gastric cancer. Carcinogenesis 34: 2452-2459, 2013.

32. Schaefer A, Jung M, Mollenkopf HJ, Wagner I, Stephan C, Jentzmik F, Miller K, Lein M, Kristiansen G and Jung K: Diagnostic and prognostic implications of microRNA profiling in prostate carcinoma. Int J Cancer 126: 1166-1176, 2010.

33. Majid S, Dar AA, Saini S, Shahryari V, Arora S, Zaman MS, Chang I, Yamamura S, Chiyomaru T, Fukuhara S, et al: MicroRNA-1280 inhibits invasion and metastasis by targeting ROCK1 in bladder cancer. PLoS One 7: e46743, 2012.

34. Gotoda T, Yamamoto H and Soetikno RM: Endoscopic submucosal dissection of early gastric cancer. J Gastroenterol 41: 929-942, 2006. 
35. Kosuke N, Oguma H and Yamamoto M: Early gastric cancer with lymph node metastasis. Ann Surg 253: 840-841, 2011.

36. Kim BS, Cho SW, Min SK and Lee BH: Differences in prognostic factors between early and advanced gastric cancer. Hepatogastroenterology 58: 1032-1040, 2011.

37. Lai IR, Lee WJ, Huang MT and Lin HH: Comparison of serum CA72-4, CEA, TPA, CA19-9 and CA125 levels in gastric cancer patients and correlation with recurrence. Hepatogastroenterology 49: 1157-1160, 2002.

38. Gao F, Chang J, Wang H and Zhang G: Potential diagnostic value of miR-155 in serum from lung adenocarcinoma patients. Oncol Rep 31: 351-357, 2014.

39. Sun Y, Wang M, Lin G, Sun S, Li X, Qi J and Li J: Serum microRNA-155 as a potential biomarker to track disease in breast cancer. PLoS One 7: e47003, 2012.
40. Chu Y, Ouyang Y, Wang F, Zheng A, Bai L, Han L, Chen Y and Wang H: MicroRNA-590 promotes cervical cancer cell growth and invasion by targeting CHL1. J Cell Biochem 115: 847-853, 2014.

41. Ma Y, Qin H and Cui Y: miR-34a targets GAS1 to promote cell proliferation and inhibit apoptosis in papillary thyroid carcinoma via PI3K/Akt/Bad pathway. Biochem Biophys Res Commun 441: 958-963, 2013

42. Meng S, Cao J, Zhang X, Fan Y, Fang L, Wang C, Lv Z, Fu D and Li Y: Downregulation of microRNA-130a contributes to endothelial progenitor cell dysfunction in diabetic patients via its target Runx3. PLoS One 8: e68611, 2013. 